\title{
Opioid risk assessment in palliative medicine
}

\author{
Rebecca Dale, DO; Jeremy Edwards, DO; and Jane Ballantyne, MD, FRCA \\ Department of Anesthesiology and Pain Medicine, University of Washington Medical Center, Seattle
}

Pain management with opioids is an integral part of palliative medicine. As the doses and durations of opioid therapy increase, the inherent risks of opioid therapy rise. Although opioids are effective analgesics, they bring with them complex medical and psychological side effects. Aberrant behavior is dangerous and can be difficult to identify as it results in a splitting in the goals of treatment between the patient and providers. One effective strategy in preventing that situation is through the early identification of at-risk patients. There are several tools that can help identify patients at higher risk of addiction and aberrant behaviors during opioid therapy. Structured use of these tools in conjunction with the clinic exam, regular follow-up visits, and lab testing can further reduce patient risk and improve success in opioid therapy. This article will review the background behind a structured strategy for opioid risk assessment using the Opioid Risk Tool, SOAPP-R, and DIRE tools. In addition, example aberrant behaviors and followup strategies will be reviewed. It will be demonstrated that careful screening and follow-up allow risk factors to be recognized and addressed early.

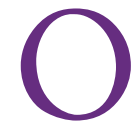
pioid therapy in palliative care requires an understanding of the tenets of palliative medicine. Palliative care does not focus solely on the dying, rather it focuses on providing comfort and improving quality of life (QoL) for any patient with life-threatening disease. The World Health Organization (WHO) describes palliative care as "an approach that improves the quality of life of patients and their families facing the problem associated with life-threatening illness, through the prevention and relief of suffering by means of early identification and impeccable assessment and treatment of pain and other problems, physical, psychosocial and spiritual."

Pain is one of the most common symptoms presented to palliative care physicians. Opioids have an established role in treating pain in the terminally ill based on their superior analgesic efficacy during short-term use. However, because of medical advances, life-threatening diseases, including cancer, are not necessarily rapidly fatal. In fact in many cases, life-threatening disease can have a long chronic phase, and may not even be the cause of death. Palliative care physicians may find themselves treating chronic pain associated with cancer disease, cancer treatment, other life-threatening illness, or incidental medical disorders. Opioids may or may not have a role in the treatment of this chronic pain. Nevertheless, it becomes increasingly important for palliative care physicians to understand and be able to identify the risks of opi- oids because they may be treating patients for years and not just months; the goals of opioid treatment may differ from end-of-life treatment goals; and the risk-benefit balance for opioids often differs at different points in the disease trajectory.

This article will focus on the necessary risk assessment that must accompany best practice use of opioid analgesics in the realm of palliative medicine. We will focus primarily on the validated tools that are available to help identify patient risk factors before initiation of opioids but will cover both medical and psychosocial risks that will affect pain control in the palliative care setting.

\section{Opioid treatment in palliative medicine: unmet needs}

In palliative medicine, pain is the most common and most distressing symptom. This is true in cancer patients in particular. In a study of about 160,000 cancer patients at the end of life, more $80 \%$ were found to have pain, and more than $60 \%$ had "very distressing pain." ${ }^{2}$ The WHO has released guidelines that recommend a stepped approach to pain treatment, starting with nonopioid analgesia and moving to opioids in a less- to more-potent progression. ${ }^{3}$ The $\mathrm{WHO}$ is one of many organizations that agree that effective pain control at the end of life may require round-the-clock opioid dosing with additional short-acting opioid for breakthrough pain. ${ }^{4,5}$

Accepted for publication December 14, 2015. Correspondence: Rebecca Dale, DO; rdale@uw.edu. Disclosures: The authors report no disclosures or conflicts of interest. JCSO 2016;14:94-100. (2) 16 Frontline Medical Communications. doi: $10.12788 /$ jcso.0229. 
Opioid therapy is a necessary component of pain control at the end of life, but primary medical teams often shy away from it. ${ }^{6}$ The involvement of a palliative medicine specialist can change this. A 2013 study showed that after a palliative care consult, patients with previously undertreated pain were more likely to be on a long-acting opioid and receive combination therapy with lower-potency adjunct medication. In addition, these patients had an average decrease of 2 points on a 10-point visual analog scale after consultation with a palliative medicine specialist. ${ }^{7}$ However, progress is not complete even within palliative medicine. A review of undertreated pain showed that more than $40 \%$ of cancer and AIDS patients reported inadequate treatment for severe pain. ${ }^{6}$ An update to that study showed that up to a third of patients with cancer still report undertreated severe pain, even with the involvement of palliative medicine specialists. ${ }^{8}$

Palliative care physicians may also find themselves treating chronic pain with opioids. Just as pain during terminal illness is often undertreated, so is chronic pain for diverse reasons. For example, the US Institute of Medicine found in 2011 that 116 million Americans had undertreated chronic pain. ${ }^{9}$ However, in a follow-up to that report, the recently published National Pain Strategy suggests a limited role for opioids in the treatment of chronic pain, and a focus on more holistic biopsychosocial approaches that minimize reliance on medications, including opioids. The difficulties palliative care physicians face when treating chronic pain in the presence of life-threatening illness are multifold: many patients are already taking opioids that were started during active disease management; the pain states are complex; prolonged use of opioids is often associated with tolerance and loss of efficacy; and it is hard to change the expectation that opioids are needed and are helpful, even when they are not.

\section{Opioid risk and its role in palliative medicine}

The question of opioid risk and how it affects decisions by the provider and the patient is not straightforward. There are many different situations, diagnoses, prognoses, goals, and wishes to consider for each patient. Some would argue that a higher level of risk is acceptable when patients have a terminal diagnosis. However, one must be careful making this assumption. Although patient comfort is of utmost importance at the end of life, the side effects of many opioid medications can have a significant impact on quality of life. These adverse effects include, but are not limited to, constipation, sedation, nausea, increased risk of falls, and dependence. Studies of terminally ill cancer patients show that many are concerned about addiction, side effects, and dose escalation. Those concerns lead patients to either decrease their pain medication or be resistant to increased doses. ${ }^{10}$ Risk of hastening a patient's death with opioid medications also influences care in palliative medicine patients.
"Double effect" refers to the concept that an increased risk of death is acceptable when the goals of care focus on the relief of severe, unmanageable pain. The acceptance of this concept is highly individualized and requires understanding and agreement from the patient and presumes that all other alternatives have been explored. ${ }^{11}$

The use of opioid therapy in palliative care is unique in that the risk-benefit ratio can vary greatly based on the patient's current clinical situation. For example, early in a patient's disease course, sedation from opioid therapy may be an unwanted side effect but the same patient may find sedation to be desirable at a later stage in their disease. The most important aspects to consider are whether the side effect will improve, worsen, is tolerable, or if it poses a significant risk. The side effects that carry the greatest risk include central nervous system toxicity (sedation, cognitive impairment, and increased risk of falls), addiction, constipation, and respiratory depression. ${ }^{12}$

Medical risks. Opioid-induced sedation is considered an adverse effect in most situations because of its often significant impairment of patient function. Sedation risks are increased when patients have underlying risk factors for falls or unsteadiness, such as advanced age or cognitive impairment. ${ }^{12}$ Patients older than 60 years who are on daily opioid therapy have a significantly increased risk of fall and fracture. Patients taking higher doses $(>50 \mathrm{mg}$ morphine equivalents/day) are twice as likely to sustain a fracture compared with those who are no longer taking opioids. ${ }^{13}$ Polypharmacy increases the risk of falls, especially with other sedating medications, such as benzodiazepines. Use of concurrent sedating medications should be carefully monitored, and lowest dose or alternate therapy should be used whenever possible. ${ }^{12}$

Opioid-induced respiratory depression is closely related to sedation and is defined as decreased ventilation after use of opioid medications. Opioid-induced sedation typically precedes opioid-induced respiratory depression and exists on the same clinical spectrum. The decrease in cortical arousal associated with opioid medications may be counteracted by stimulus such as pain, but this effect cannot be counted on in chronic pain. ${ }^{14}$ Respiratory depression has been shown to be dose related, with mortality at three times higher at $>200 \mathrm{mg}$ morphine equivalent dose. ${ }^{15}$ Like sedation, polypharmacy with other sedating medications will increase the risk of respiratory depression and mortality. Patients on multiple sedating medications, with underlying respiratory disease, or on very high doses of opioids should be monitored closely as they can be considered high-risk patients. ${ }^{12,16}$

Opioid-induced constipation is an adverse effect of opioids for which tolerance does not develop. It is experienced by almost all patients because of the mu-agonist effects of opioids in the gut. However, constipation is often underreported and therefore undertreated. A study of hospice 
patients showed that all patients experienced constipation, but it was only rarely noted in their nursing notes. ${ }^{17}$ Like other opioid adverse effects, concurrent use of additional medications, such as anticholinergics and chemotherapy agents, can have an additive effect on constipation. ${ }^{12}$ The stool habits in any patient on opioid medications, regardless of dose or length of treatment, should be carefully monitored. Bowel management regimens should be initiated simultaneously with opioid therapy. No single bowel regimen has been shown to be more effective than any other, the key to treatment is early introduction. ${ }^{16}$

Addiction and aberrant behavior. It has been well established that patients with a personal or family history of substance use disorder, history of childhood abuse, or history of mental illness are more likely to engage in aberrant drug-related behavior. ${ }^{18}$ In addition, patients with substance use disorder tend to be more complex and require more management than patients who do not have the same comorbidities. ${ }^{19}$ It is imperative that providers determine who is at increased risk of addiction and aberrant behavior before they prescribe opioid therapies. Untreated addiction and substance abuse problems in palliative care patients comes with many inherent adverse effects: increased suffering, complicated treatment and management, endangered patient safety, and weakened social supports. ${ }^{20} \mathrm{~A}$ related concern is drug diversion. This can involve patients, family members, or caregivers selling medications for profit or sharing medication with others for recreational use. The large doses of high potency opioids often required in palliative care can be a significant temptation to patients and families. In addition, the presence of substance abuse or other psychiatric illnesses are significantly associated with other concerns such as decreased efficacy of primary treatments and reduced provider-patient trust. ${ }^{21}$

\section{Opioid risk assessment}

Risk assessment should be formally integrated into every aspect of the patient's medical care. Specifically, risk assessment should be completed before making any treatment decisions regarding opioid therapy. It must be noted that the intake risk assessment is a single point in time that can easily change as therapy progresses. Ongoing risk assessment through the course of treatment is crucial to ensure
TABLE 1 Validated risk factors for developing addiction with opioid

History of substance abuse, including smoking

Family history of substance abuse

History of abuse, particularly childhood abuse and post-traumatic stress disorder

Psychiatric comorbidities (especially depression, anxiety, and personality disorders

adequate opioid safety and effectiveness of care. ${ }^{22}$ By risk stratifying patients with a validated clinical tool, providers can ensure that patients who are at higher risk of abuse and aberrant behavior have access to additional safety measures that are integrated into their treatment plans.

A thorough and detailed assessment must be completed anytime a patient is being considered for opioid therapy. Using a standardized format for assessment will decrease variability among providers and allow for a more consistent assessment throughout treatment. A complete history and physical exam should also be taken. This includes a review of the medical records and prior pain treatment, if any. This will give the provider an overview of the analgesic needs of the patient and may shed light on prior aberrant behavior. ${ }^{23}$ However, as these topics are often sensitive in nature, they may not be clearly stated in medical documentation. A clinician must be adept at "reading between the lines" to obtain the full story. During the medical record review, the clinician should be aware of the presence of any of the clinically validated risk factors for developing addiction as mentioned previously (Table 1). ${ }^{12}$ In addition to the structured medical interview and review of the chart, clinicians should use a validated screening tool to assist them with risk assessment. These tools are developed for clinical use as they generally are brief and can be either self-administered or quickly administered by a provider during an office visit. One of the more commonly used tools, the Opioid Risk Tool (ORT), has 8 questions and has been validated in both cancer and noncancer pain. ${ }^{24,25}$ Another self-administered tool is the Screener and Opioid Assessment for Patients

TABLE 2 Recommended screening tools

\section{Tool}

Opioid Risk Tool (ORT) ${ }^{19}$

Screener and Opioid Assessment for Patients with Pain Revised (SOAPP-R) ${ }^{11}$

Diagnosis, Intractability, Risk, Efficacy $\left(\right.$ DIRE$^{31}$

\section{Description}

5 items, self-performed

14 items (revised) and 5 items (short form), self-performed

7 items, staff performed

\section{Used for}

Better for low-risk screening

Determines needs for increased monitoring during therapy. Useful for higher-risk populations.

Primary care tool for risk of opioid abuse 
with Pain-Revised (SOAPP-R), which has been validated in large groups of noncancer pain patients. ${ }^{26,27}$ SOAPP-R is a 24-item self-reported list with good clinical applicability. ${ }^{26}$ The Diagnosis, Intractability, Risk and Efficacy inventory (DIRE) is a staff-administered tool that scores a patient in 4 categories, each of which contributes to the overall risk score (Table 2). The Risk category is split into subcategories of psychological, chemical health, reliability, and social support. A staff member who is familiar with the patient can complete the tool in a few minutes. ${ }^{18}$

When compared head to head, each tool has its own strengths and weaknesses. A small study showed that the sensitivities of the clinical interview, SOAPP-R, ORT, and DIRE were $0.77,0.73,0.45$, and 0.17 , respectively. The study concluded that although the DIRE and ORT did not have the same sensitivity as the SOAPP-R, they had specific circumstances in which they improved. The DIRE tool was found to have increased sensitivity (0.36) in patients with multiple episodes of aberrant behavior, indicating it may be of more use in the higher-risk populations as a follow-up tool. The ORT tool was thought to be most useful in large-volume practices because it is easy to use. However, given concerns about patient manipulation of the ORT, we suggest its use for a lower-risk population in which the tendency to mislead is lower. When combined with a thorough clinical interview, the SOAPP-R sensitivity to identifying individuals at risk of addition behaviors approaches 0.90 , making it very effective. ${ }^{18}$

Pre-existing addiction and substance abuse is a common finding in patients with chronic pain. A study of palliative medicine patients who had been referred to a pain clinic found that at the time of initial interview, $46 \%$ had a positive SOAPP screen and $15 \%$ had a positive CAGE screen for alcohol abuse. ${ }^{28}$ Pediatric patients are not immune to this concern. A study from a pediatric cancer center showed that more than $11 \%$ of adolescent and young adult cancer patients prescribed opioids demonstrated aberrant opioid misuse behaviors. ${ }^{29}$ The discussion of pre-existing substance abuse disorder or opioid misuse and aberrant behavior is a difficult but important conversation.

\section{Management of addiction risk}

After a patient has been placed into a risk category based on a validated tool, treatment can begin. A consistent, step-wise approach to prescribing and managing opioid medication in the palliative care patient population is recommended. Aspects of best practice opioid management include informed consent, treatment agreements, pre- and post-intervention assessments of pain and function, use of adjuncts, regular review of pain diagnoses and comorbidities, regular review of addiction potential, and careful documentation of all aspects of management. ${ }^{23}$

During the frequent reassessments, providers should be aware of red flag aberrant behaviors that can indicate addic-

\section{TABLE 3 Aberrant behaviors}

$$
\text { Forged prescriptions }
$$

Doctor shopping

$$
\text { Urine drug testing aberrancies }
$$

Lost opioid prescriptions

\section{Requesting early prescriptions}

Unscheduled clinic visits due to pain complaints

Emergency room visits due to pain complaints

Requests for opioid dose escalation

tion. These behaviors include requests for dose escalation, emergency room visits, repeatedly seeking early prescription fills, doctor shopping, and forged prescriptions. ${ }^{25}$ Not all aberrant behaviors are cause for concern. For example, if a patient is requesting early prescription refills, it is important to distinguish between escalating tolerance, escalating pain, and addiction. One should take a step back and evaluate if dose escalation is the answer or if other approaches could be used more effectively.

Urine drug screening. This is an important addition to written screening tools during management of opioids. It can be useful in identifying illicit substances use or nonuse of prescribed substances. A negative drug screen for a prescribed opioid may indicate diversion..$^{31} \mathrm{~A}$ baseline urine drug screen to identify possible preexisting drug abuse is strongly advised. Thereafter, drug screening can be done for cause or regularly, according to clinic protocol. Some providers recommend repeat drug screening at least annually. Both screening urine tests and urine drug testing with confirmation are used in evaluations during opioid management. Screening urine testing uses antibody reagents to test for the presence of substances in the urine. Wide availability and ease of performing the test allows these screening urine tests to be completed by office staff at the time of the patient visit. It is generally accepted that urine screening and confirmatory testing are appropriate for first-line screening and testing. ${ }^{12,16,32}$ However, additional screening methods, such as screening hair, blood, or saliva are also available and can be used if urine testing is not appropriate or available. Immunoassay screening urine tests do not reliably distinguish between different opioids so are not helpful in a patient taking an illicit opioid. In contrast, confirmatory urine drug screens can use gas chromatography and mass spectroscopy to identify specific substances found in the patient's urine. ${ }^{33}$

The specificity of a confirmatory test allows precise monitoring of the patient in the hands of an experienced palliative care clinician. However, the cost of confirmatory 


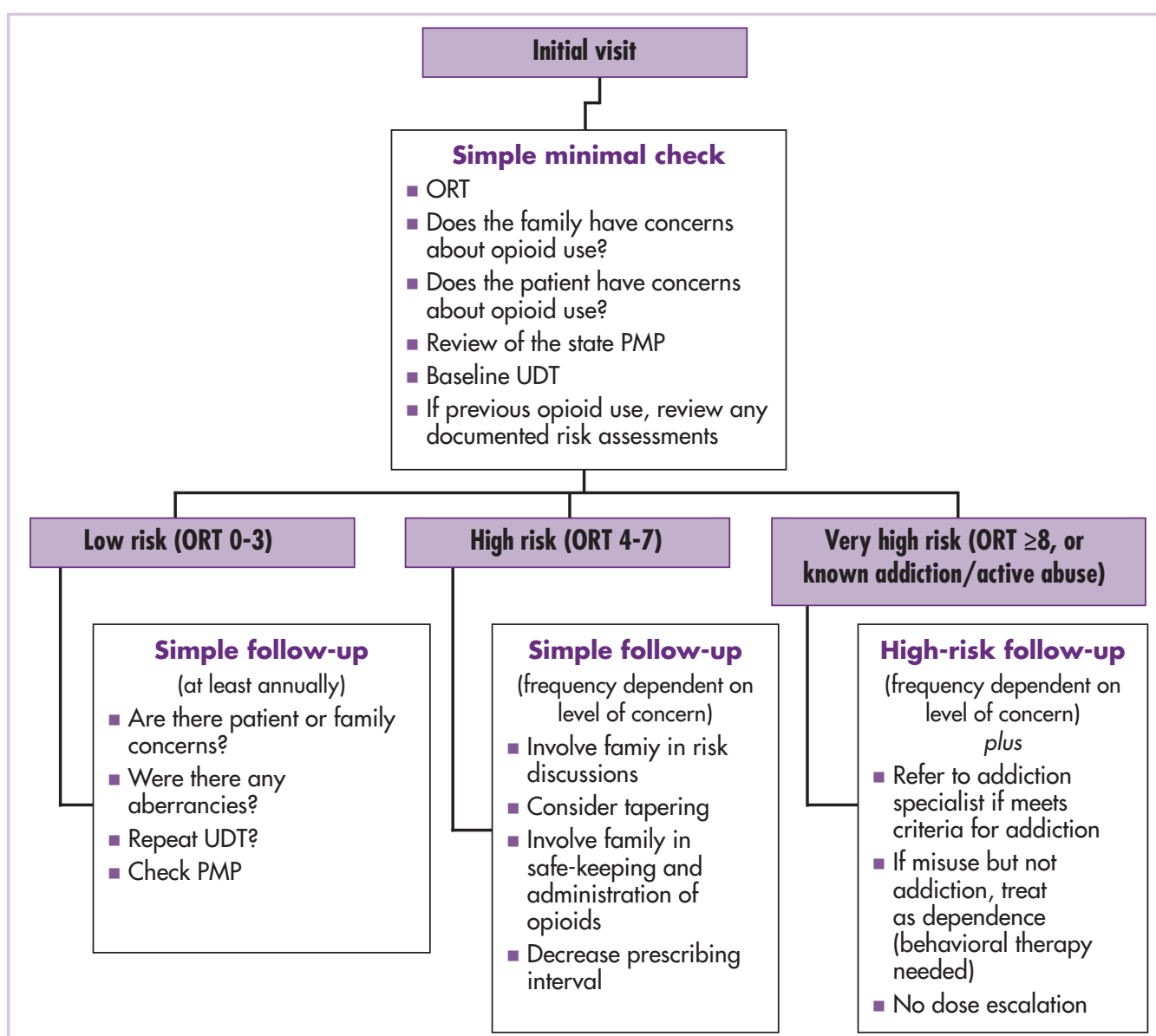

FIGURE Process algorithm for assessing opioid risk both initially and in ongoing treatment in patients with pain in the setting of palliative medicine. ${ }^{a}$

ORT, Opioid Risk Tool; PMP, Prescription Monitoring Program; UDT, urine drug testing

aFor inpatient or hospice short-term therapy, opioids are given under supervision and as needed. Apply usual safety precautions.

pliance methods, such as prescription monitoring programs and urine drug testing. ${ }^{34}$

Written treatment agreement. An additional tool for opioid management is a written treatment agreement between the patient and the provider. The agreement should clearly delineate the roles of the provider and the patient during the opioid treatment course. Treatment agreements can be especially helpful when aberrant behaviors emerge and consequences need to be determined. Changes to therapy, such as more frequent follow-up and short-duration prescriptions can be helpful in the face of aberrant behavior by ensuring that large quantities of opioids are not available to the patient. In addition, use of a prescription-monitoring program will decrease the incidence of doctor shopping and allow for more consistent follow-up and monitoring. ${ }^{12,35}$ During opioid therapy, the involvement of family and friends is very helpful in successful monitoring for aberrant behavior and opioid misuse. Patient self-reporting may be unreliable for a wide variety of reasons. A third-party reporter,

testing is higher and the tests are not readily available in an office setting. Therefore, screening urine immunoassay testing with selective use of confirmatory testing is considered the most reasonable approach. ${ }^{12}$ The level of testing will depend on clinical judgment of the level of concern. All types of urine drug screening need to be interpreted in the context of each patient's individual clinical situation. A positive urine drug test cannot be viewed in isolation. Accurate interpretation of both screening and confirmatory testing requires a clear understanding of opioid pharmacokinetics and metabolism and should be done by a provider who is qualified to do so. ${ }^{16}$ If the results of a urine test are unexpected, of concern, or ambiguous it is often very helpful to speak to the lab for further clarification. Additional compliance measures, such as random pill count and patch count can also be used, but they are limited in their usefulness because they cannot account for surplus medication supply or as-needed dosing schedules. For that reason, pill or patch counts are most useful as an adjunct to other com- such as a family member can help to ensure providers have accurate information regarding risk assessment. Family members must be educated early in the opioid prescription and monitoring process to ensure they are aware of risks and responsibilities. ${ }^{12,16}$

\section{Opioids in palliative care: further considerations}

The increasing prevalence of opioid use in both palliative medicine and chronic pain treatment has highlighted the risks of chronic opioid use. However, the choice to prescribe opioids for both cancer and noncancer pain is not a simple question of "to prescribe or to not prescribe." The risk-benefit analysis is not at all straightforward and is different for each patient. The decision should be centered on the wishes and concerns of the patient and the patient's family, with guidance from clinicians about risk and benefits, and how the risk can be managed. It is not uncommon for patients to desire less therapy so that side effects such as sedation may be avoided. In a study of more than 
1,100 cancer patients, more than $80 \%$ reported not taking additional pain medication in order to avoid side effects. ${ }^{37}$ Patients have very strong preferences regarding their symptomatic care and individual methods for managing their pain. When a patient shows aberrant behavior, the palliative medicine clinician has a responsibility to ensure that additional support and alternative methods are used to reduce risk and increase patient safety. ${ }^{36}$

\section{Opioid risk assessment: a process algorithm}

The high risk of opioid medications must be considered when treating patients with pain in the setting of palliative medicine. However, with careful screening and information gathering, safe and effective pain control can be achieved in this complex population of patients (Figure).

\section{Conclusion}

Opioid analgesics are now a common and standard therapy in palliative medicine. As patients are living longer, doses and duration of opioid therapies are increasing significantly. Accordingly, the risks of opioid therapy have

\section{References}

1. World Health Organization. National cancer control programmes: policies and managerial guidelines http://www.who.int/cancer/publications/nccp2002/en/. Published 2002. Accessed Janaury 22, 2016.

2. Costantini M, Ripamonti C, Beccaro M, et al. Prevalence, distress, management, and relief of pain during the last 3 months of cancer pa life. Results of an Italian mortality follow-back survey. Ann Oncol. 2009;20:729-735.

3. World Health Organization. Cancer pain relief: with a guide to opioid availability (2nd ed). Geneva: World Health Organization; 1996.

4. Ripamonti CI, Santini D, Maranzano E, Berti M, Roila F, Group EGW. Management of cancer pain: ESMO Clinical Practice Guidelines. Ann Oncol. 2012;23(suppl 7):vii139-54.

5. Caraceni A, Hanks G, Kaasa S, et al. Use of opioid analgesics in the treatment of cancer pain: evidence-based recommendations from the EAPC. Lancet Oncol. 2012;13:e58-68.

6. Deandrea S, Montanari M, Moja L, Apolone G. Prevalence of undertreatment in cancer pain. A review of published literature. Ann Oncol.2008;19:1985-1991.

7. Muir J, Scheffey C, Young H. Opioid prescribing practices before and after initiation of palliative care in outpatients. J Pain Symptom Manage. 2013;45:1107-1111.

8. Greco MT, Roberto A, Corli O, et al. Quality of cancer pain management: an update of a systematic review of undertreatment of patients with cancer. J Clin Oncol. 2014;32:4149-4154.

9. Institute of Medicine. Relieving pain in America. A blueprint for transforming prevention, care, education, and research. http:// www.nap.edu/read/13172/chapter/1\#ix . Published 2011. Accessed January 22, 2016.

10. Weis S, Emanuel L, Fairclough D. Understanding the experience of pain in terminally ill patients. Lancet. 2001;357:1311-1315.

11. Regnard C, George R, Grogan E, et al. So, farewell then, doctrine of double effect. Br Med J. 2011;343:d4512.

12. Krashin D, Murinova N, Jumelle P, Ballantyne J. Opioid risk assessment in palliative medicine. Expert Opin Drug Saf 2015;14:1023-1033.

13. Saunders KW, Dunn KM, Merrill JO, et al. Relationship of opioid use and dosage levels to fractures in older chronic pain patients. J Gen Intern Med. 2010;25:310-315.

14. Boland J, Boland E, Brooks D. Importance of the correct diagnosis of opioid-induced respiratory depression in adult cancer patients and also increased. Although the risks vary from patient to patient, it can be held that the risks of adverse effects increases as doses increase. Opioids have numerous medical adverse effects such as sedation, constipation, and respiratory depression, but these are often easily identified and treated. However, the risk of addiction and aberrant behavior can be difficult to identify and can greatly complicate opioid therapy. Using established and validated screening tools such as the ORT and SOAPP-R can help to identify at-risk patients before initiation of therapy, allowing clinicians to create individual treatment regimens that mitigate risk and still provide adequate symptom management. Once a treatment plan has been established and initiated, frequent follow-up is crucial for all patients regardless of risk stratification. Less frequent follow-up is necessary in low-risk patients, but rescreening, intermittent urine drug screening, and observation for red flags for addiction is still required. High-risk patients require more frequent visits and observation in addition to more frequent use of confirmatory urine drug testing. titration of naloxone. Clin Med. 2013;13:149-151.

15. Gomes T, Mamdani MM, Dhalla IA, Paterson JM, Juurlink DN. Opioid dose and drug-related mortality in patients with nonmalignant pain. Arch Intern Med. 2011;171:686-691.

16. Chou R, Fanciullo GJ, Fine PG, et al. Clinical guidelines for the use of chronic opioid therapy in chronic noncancer pain. J Pain. 2009;10:113-130.

17. McMillan SC, Tittle M. A descriptive study of the management of pain and pain-related side effects in a cancer center and a hospice. Hosp J. 1995;10:89-107.

18. Moore TM, Jones T, Browder JH, Daffron S, Passik SD. A comparison of common screening methods for predicting aberrant drugrelated behavior among patients receiving opioids for chronic pain management. Pain Med. 2009;10:1426-1433.

19. Bruera E. Ethical issues in palliative care research. J Palliat Care. 1994;10:7-9.

20. Passik SD, Theobald DE. Managing addiction in advanced cancer patients: why bother? J Pain Symptom Manage. 2000;19:229-234.

21. Passik SD, Portenoy RK, Ricketts PL. Substance abuse issues in cancer patients. Part 1: Prevalence and diagnosis. Oncology (Williston Park). 1998;12:517-521, 524.

22. Reuben DB, Alvanzo AA, Ashikaga T, et al. National Institutes of Health Pathways to Prevention Workshop: the role of opioids in the treatment of chronic pain. Ann Intern Med. 2015;162:295-300.

23. Gourlay DL, Heit HA, Almahrezi A. Universal precautions in pain medicine: a rational approach to the treatment of chronic pain. Pain Med. 2005;6:107-112.

24. Ma JD, Horton JM, Hwang M, Atayee RS, Roeland EJ. A singlecenter, retrospective analysis evaluating the utilization of the opioid risk tool in opioid-treated cancer patients. J Pain Palliat Care Pharmacother. 2014;28:4-9.

25. Webster LR, Webster RM. Predicting aberrant behaviors in opioidtreated patients: preliminary validation of the Opioid Risk Tool. Pain Med. 2005;6:432-442.

26. Butler SF, Budman SH, Fernandez KC, Fanciullo GJ, Jamison RN. Cross-Validation of a Screener to Predict Opioid Misuse in Chronic Pain Patients (SOAPP-R). J Addict Med. 2009;3:66-73.

27. Butler SF, Fernandez K, Benoit C, Budman SH, Jamison RN. Validation of the revised Screener and Opioid Assessment for Patients with Pain (SOAPP-R). J Pain. 2008;9:360-372. 


\section{How We Do it}

28. Childers JW, King LA, Arnold RM. Chronic pain and risk factors for opioid misuse in a palliative care clinic. Am J Hosp Palliat Care. 2015;32:654-659.

29. Ehrentraut JH, Kern KD, Long SA, An AQ, Faughnan LG, Anghelescu DL. Opioid misuse behaviors in adolescents and young adults in a hematology/oncology setting. J Pediatr Psychol. 2014;39:1149-1160.

30. Passik SD, Kirsh KL, Webster L. Pseudoaddiction revisited: a commentary on clinical and historical considerations. Pain Manag. 2011;1:239-248

31. Christo PJ, Manchikanti L, Ruan X, et al. Urine drug testing in chronic pain. Pain Physician. 2011;14:123-143.

32. Passik SD, Squire P. Current risk assessment and management paradigms: snapshots in the life of the pain specialist. Pain Med. 2009;10(suppl 2):S101-14.

33. Manchikanti L, Malla Y, Wargo BW, Fellows B. Comparative evaluation of the accuracy of immunoassay with liquid chromatogra- phy tandem mass spectrometry (LC/MS/MS) of urine drug testing (UDT) opioids and illicit drugs in chronic pain patients. Pain Physician. 2011;14:175-187.

34. Lam WY, Fresco P. Medication adherence measures: an overview. Biomed Res Int. 2015. doi: 10.1155/2015/217047.

35. Passik SD, Portenoy RK, Ricketts PL. Substance abuse issues in cancer patients. Part 2: Evaluation and treatment. Oncology (Williston Park). 1998;12:729-734; discussion 36, 41-2.

36. Anghelescu DL, Ehrentraut JH, Faughnan LG. Opioid misuse and abuse: risk assessment and management in patients with cancer pain. J Natl Compr Canc Netw. 2013;11:1023-1031.

37. Simone CB, 2nd, Vapiwala N, Hampshire MK, Metz JM. Cancer patient attitudes toward analgesic usage and pain intervention. Clin J Pain. 2012;28:157-162.

38. Belgrade MJ, Schamber CD, Lindgren BR. The DIRE score: predicting outcomes of opioid prescribing for chronic pain. J Pain. 2006;7:671-681 